\title{
Comparison of right ventricle-pulmonary artery shunt position in the Single Ventricle Reconstruction trial
}

\author{
Nicholas D. Andersen, MD, ${ }^{a}$ James M. Meza, MD, ${ }^{\text {a,b,e }}$ Matthew R. Byler, MD, ${ }^{c}$ Andrew J. Lodge, MD, ${ }^{a}$ \\ Kevin D. Hill, MD, MS, ${ }^{\text {de }}$ Christoph P. Hornik, MD, MPH, ${ }^{\mathrm{d}, \mathrm{e}}$ and Robert D. B. Jaquiss, MD
}

\section{ABSTRACT}

Objective: Placement of a right ventricle-pulmonary artery shunt to the left or right of the neoaorta may influence reinterventions, pulmonary artery development, and survival after the Norwood procedure because of differences in shunt and pulmonary artery geometry and blood flow.

Methods: We analyzed the Pediatric Heart Network Single Ventricle Reconstruction Trial public use dataset. Comparisons were made between patients who received a left- or right-sided right ventricle-pulmonary artery shunt during the Norwood procedure in both the overall $(n=274)$ and the propensity scorematched (67 pairs) patient cohorts.

Results: A left-sided shunt was placed in 168 patients $(61 \%)$, and a right-sided shunt was placed in 106 patients $(39 \%)$ ). At the 12-month follow-up, there were no differences in pulmonary artery measurements, hemodynamic measurements, or pulmonary artery reinterventions between shunt groups. However, the rightsided shunt was associated with fewer surgical shunt revisions in both the overall (8.3 vs 1.9 events per 100 infants, $P=.05$ ) and the propensity score-matched $(17.9$ vs 0 events per 100 infants, $P<.001)$ patient cohorts. In the propensity score-matched cohort only, right-sided shunts were further associated with fewer serious adverse events (84 vs 46 events per 100 infants, $P=.01$ ) and improved transplantation-free survival at 3 years follow-up $(61 \%$ [95\% confidence interval, 48-72] vs $80 \%$ [95\% confidence interval, 69-88], $P=.04)$.

Conclusions: In the Single Ventricle Reconstruction trial, right ventricle-pulmonary artery shunt placement to the right of the neoaorta was associated with fewer shunt revisions and may contribute to improved outcomes in select patients. ( $\mathrm{J}$ Thorac Cardiovasc Surg 2017;153:1490-500)

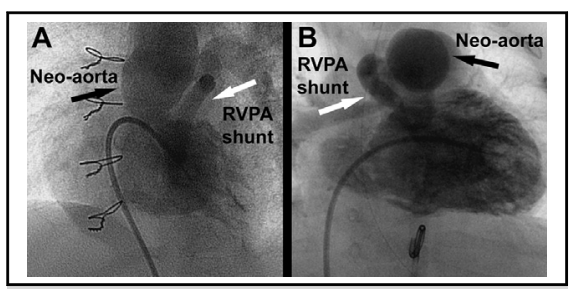

RVPA shunt positions in the Norwood procedure.

Central Message

RVPA shunt placement to the right of the neoaorta was associated with fewer surgical shunt revisions in the SVR trial.

\section{Perspective}

In the SVR trial, RVPA shunts led to improved transplantation-free survival but more unintended interventions and worse branch PA growth. This secondary analysis of the SVR trial found that RVPA shunt reinterventions may be reduced by placement of the RVPA shunt to the right of the neoaorta.

See Editorial Commentary page 1501.
The outcomes of stage I reconstruction (Norwood procedure) for patients with hypoplastic left heart syndrome have gradually improved over time, in part because of incremental advances in surgical technique. ${ }^{1}$ The landmark

From the a Division of Cardiovascular and Thoracic Surgery, Department of Surgery, and ${ }^{\mathrm{d}}$ Division of Pediatric Cardiology, Department of Pediatrics, Duke University Medical Center; and ${ }^{\mathrm{e}}$ Duke Clinical Research Institute, Durham, NC; ${ }^{\mathrm{b}} \mathrm{Hospital}$ for Sick Children, The Congenital Heart Surgeons' Society Data Center, Toronto, Ontario, Canada; and ${ }^{\mathrm{c} D e p a r t m e n t}$ of Surgery, University of Virginia, Charlottesville, Va

ClinicalTrials.gov number: NCT00115934

Read at the 42nd Annual Meeting of The Western Thoracic Surgical Association, Waikoloa, Hawaii, June 22-25, 2016.

Received for publication June 21, 2016; revisions received Oct 12, 2016; accepted for publication Oct 12, 2016; available ahead of print March 31, 2017.

Address for reprints: Nicholas D. Andersen, MD, Division of Cardiovascular and Thoracic Surgery, Duke University Medical Center, Box 3443, Durham, NC 27710 (E-mail: Nicholas.Andersen@Duke.edu).

0022-5223/\$36.00

Copyright (c) 2017 by The American Association for Thoracic Surgery

http://dx.doi.org/10.1016/j.jtcvs.2016.10.104
Pediatric Heart Network Single Ventricle Reconstruction (SVR) trial evaluated the source of pulmonary blood flow in patients undergoing the Norwood procedure and demonstrated that use of right ventricle-pulmonary artery (RVPA) shunts led to improved early survival compared with modified Blalock-Taussig (MBT) shunts, but came at the expense of more unintended interventions and worse branch pulmonary artery (PA) growth. ${ }^{2,3}$ However, numerous technical aspects of RVPA shunt placement, such as shunt size, position, choice of conduit, and anastomotic technique, may further

Scanning this QR code will take you to supplemental figure and table for this article. 


\section{Abbreviations and Acronyms \\ $\mathrm{CPB}=$ cardiopulmonary bypass \\ L-RVPA = left-sided right ventricle-pulmonary artery \\ MBT = modified Blalock-Taussig \\ PA $=$ pulmonary artery \\ $\mathrm{R}-\mathrm{RVPA}=$ right-sided right ventricle-pulmonary artery \\ RVPA = right ventricle-pulmonary artery \\ SVR $=$ Single Ventricle Reconstruction}

influence these results. One potentially important technical variable that was recorded, but not dictated, by the SVR trial protocol was placement of the RVPA shunt to the left or right of the neoaorta. Although the shunt was originally routed to the left of the neoaorta, several authors have advocated for rightward placement of the RVPA shunt..$^{4-7}$ Purported benefits of right-sided (R)-RVPA shunt placement include (1) reduced kinking of the shunt leading to fewer shunt reinterventions; (2) reduced tension and more central placement on the PAs leading to more symmetric PA blood flow, improved PA growth, and fewer PA reinterventions; and (3) greater ease of surgical access to the distal shunt and central PAs during the stage II procedure leading to improved stage II outcomes. Limited single-institution reports have supported some of these assertions and demonstrated improved oxygen saturations, ${ }^{7}$ improved PA growth, ${ }^{6,7}$ decreased PA reinterventions, ${ }^{6}$ decreased cardiopulmonary bypass (CPB) and ischemic times at stage II operation, ${ }^{5}$ and even improved survival with R-RVPA shunts. ${ }^{5}$ We aimed to validate these findings and compare outcomes between patients who received a left-sided (L)-RVPA or R-RVPA shunt in the SVR trial dataset, which represents the largest multicenter, prospective cohort of infants with longitudinal followup after the Norwood procedure. We hypothesized that R-RVPA shunt placement would be associated with superior outcomes.

\section{MATERIALS AND METHODS Study Design}

The SVR trial was conducted between May 2005 and July 2008 at 15 North American centers and randomized 549 patients with hypoplastic left heart syndrome or related single right ventricle lesions to receive an RVPA shunt or MBT shunt at the time of the Norwood procedure. Details of the trial design and main results have been reported. ${ }^{2,8}$ The Institutional Review Board at each participating center approved the trial (ClinicalTrials.gov number, NCT00115934), and written informed consent was obtained from 1 or both parents. The National Institutes of Health/National Heart, Lung, and Blood Institute Pediatric Heart Network SVR Trial public use dataset was used exclusively for the preparation of this work. Data were downloaded from https://www. pediatricheartnetwork.com/pud_login.asp?study_id=SVR on November
23, 2015. Only patients who received an RVPA shunt at the conclusion of the Norwood procedure were included in the analysis.

\section{Definitions and Study Measurements}

For patients who received an RVPA shunt at the conclusion of the Norwood procedure, the position of the shunt relative to the neoaorta (left or right) was recorded on the Supplemental Data Form (variable code RVPAS_SIDE). All other baseline, operative, and outcomes variables were reported as described in prior publications., ${ }^{2,3,8,9}$ Center and surgeon yearly Norwood procedure volume estimates were based on the number of screened patients. ${ }^{2,8}$ Serious adverse events were defined as death, acute shunt failure, cardiac arrest, extracorporeal membrane oxygenation, unplanned cardiovascular reoperation, or necrotizing enterocolitis. $^{2,8}$ Complications included any postoperative cardiac, respiratory, neurologic, gastrointestinal, infectious, renal, hematologic, vascular, or other complications as defined on the "PHN Code List M Complications" data form. Phrenic nerve injury/diaphragm paralysis was defined as newly elevated diaphragm on chest x-ray. Vocal cord injury was defined as vocal cord immobility on direct visualization. Hemodynamic and angiographic measurements from interpretable prestage II catheterizations were performed by the Angiography Core Laboratory, and no additional independent angiographic review was performed for this study. The Nakata index (also called the "PA index") was used to estimate central PA growth from mid-main branch PA measurements and was calculated using the formula $\pi \times$ [mid-main right PA diameter $(\mathrm{mm})^{2}+$ mid-main left PA diameter $\left.(\mathrm{mm})^{2}\right] /[4 \times$ body surface area $\left(\mathrm{m}^{2}\right)$ ]. The lower lobe index was used to estimate peripheral PA growth from the proximal lower lobe branch PA (after the takeoff of the upper lobe branch) and was calculated using the formula $\pi \times$ [right lower lobe branch PA diameter $(\mathrm{mm})^{2}+$ left lower lobe branch PA diameter $\left.(\mathrm{mm})^{2}\right] /\left[4 \times\right.$ body surface area $\left.\left(\mathrm{m}^{2}\right)\right] . .^{3,10,11}$ The severity of branch PA stenosis was quantified as none $(<15 \%)$, mild $(15 \%-35 \%)$, moderate $(>35 \%-50 \%)$, or severe $(>50 \%)$, as described by Aiyagari and colleagues. ${ }^{3}$

\section{Statistical Methods}

All comparisons were made between patients who received an L-RVPA or R-RVPA shunt at the conclusion of the Norwood procedure regardless of the assigned treatment group, representing a non-intention-to-treat analysis. Although baseline characteristics were similar between shunt groups, several important operative parameters differed between groups. As a result, patients were propensity matched to produce 2 patient cohorts with a similar propensity for receiving an L-RVPA or R-RVPA shunt. Propensity scores for receiving an L-RVPA or R-RVPA shunt were calculated by multivariable conditional logistic regression including the following covariates: sex, birth weight, gestational age, prenatal diagnosis, any associated anatomic diagnosis, age at Norwood procedure, year of operation, low-volume center ( $\leq 15$ cases per year), shunt diameter, CPB time, crossclamp time, and perfusion strategy. Patients were matched on propensity scores within a 0.1 margin using the greedy match algorithm in a 1:1 ratio. The model produced 2 groups with a similar propensity for receiving an L-RVPA or R-RVPA shunt (Figure E1). Matching covariates and standardized difference of the mean are shown in Table E1.

Summary data are presented as median with interquartile range or frequency counts with percentages. Reinterventions and adverse events are reported as the event rate per 100 enrolled infants, consistent with prior SVR trial publications. ${ }^{2}$ Continuous and categoric variables were compared using the Mann-Whitney rank-sum test or the Fisher exact test, respectively. Reintervention and adverse event rates were compared using the Poisson means test. Overall transplantation-free survival was compared between groups using Kaplan-Meier curves and the GehanWilcoxon test. Calculations were performed using STATA 11.1 (StataCorp LP, College Station, Tex) and SAS 9.2 (SAS Institute Inc, Cary, NC). 


\section{RESULTS \\ Baseline Characteristics}

A total of 281 patients in the SVR trial underwent RVPA shunt placement during the Norwood procedure; the position of the shunt relative to the neoaorta was recorded for 274 patients $(98 \%)$ who comprised the final study cohort. A left-sided shunt was placed in 168 patients $(61 \%)$, and a right-sided shunt was placed in 106 patients $(39 \%)$. The proportion of R-RVPA shunts remained nearly constant from year to year $(35 \%$ in $2005,43 \%$ in $2006,36 \%$ in $2007,41 \%$ in 2008), suggesting no change in surgeon preference for shunt position over the course of the study. Although RVPA shunt position was not randomized, baseline characteristics remained well balanced, with no significant differences between groups (Table 1).

\section{Operative Parameters}

Operative variables for patients who underwent RVPA shunt placement during the stage 1 procedure $(n=274)$, as well as those who progressed to undergo the stage II procedure $(n=219)$, are shown in Table 2. Differences in the conduct of surgery were appreciated between groups. LRVPA shunts were used more commonly by high-volume centers and surgeons, and were associated with shorter CPB and crossclamp times and more frequent use of deep hypothermic circulatory arrest (DHCA) as the perfusion strategy. Shunt diameters also differed between groups (L-RVPA: 68\% $5 \mathrm{~mm}, 26 \% 6 \mathrm{~mm}$; R-RVPA: 46\% $5 \mathrm{~mm}$, $52 \% 6 \mathrm{~mm}$ ), with 6-mm shunts used more often in the RRVPA group.
As a result of the differences in operative parameters, which could confound the comparison of outcomes between groups, a subgroup analysis was performed using propensity score matching to produce 2 patient cohorts $(n=67$ patients per group) with a similar propensity for receiving an L- or R-RVPA shunt. In the propensity score-matched cohort, baseline characteristics and key operative parameters, including center volume, surgeon volume, shunt size, CPB time, crossclamp time, and perfusion strategy, were well balanced between groups. The only statistically significant difference between propensity score-matched patients was that patients in the R-RVPA shunt group weighed less at birth (Tables 1 and 2).

\section{Pulmonary Artery and Shunt Reinterventions}

Unintended PA and shunt interventions before 12 months of age are summarized in Table 3. There were no differences in the rate of PA interventions between L-RVPA and RRVPA groups in the overall or propensity score-matched cohorts. The majority of PA interventions $(72 \%, 79$ of 110 PA interventions) entailed pulmonary arterioplasty at the time of stage II operation.

The total rate of unintended shunt intervention was $67 \%$ higher with L-RVPA shunts (22 events vs 13.2 events per 100 infants), but this difference failed to reach statistical significance $(P=.12)$. However, the rate of surgical shunt revision was 4.4-fold higher with L-RVPA shunts compared with R-RVPA shunts (8.3 vs 1.9 events per 100 infants, $P=.05$ ). In the overall cohort, 12 patients underwent 16 shunt revisions before stage II operation. Mortality for

TABLE 1. Baseline characteristics

\begin{tabular}{|c|c|c|c|c|c|c|}
\hline \multirow[b]{2}{*}{ Variable } & \multicolumn{3}{|c|}{ Total cohort } & \multicolumn{3}{|c|}{ Propensity score-matched cohort } \\
\hline & $\begin{array}{l}\text { L-RVPAS } \\
(n=168)\end{array}$ & $\begin{array}{l}\text { R-RVPAS } \\
(\mathbf{n}=106)\end{array}$ & $P$ value & $\begin{array}{c}\text { L-RVPAS } \\
(\mathrm{n}=67)\end{array}$ & $\begin{array}{c}\text { R-RVPAS } \\
(\mathbf{n}=67)\end{array}$ & $P$ value \\
\hline Male sex & $108(64 \%)$ & $61(58 \%)$ & .26 & $41(61 \%)$ & $39(58 \%)$ & .73 \\
\hline Birth weight $(\mathrm{kg})$ & $3.1[2.7-3.4]$ & $3.1[2.8-3.4]$ & .88 & $3.3[2.9-3.7]$ & $3.0[2.7-3.4]$ & .02 \\
\hline Birth weight $<2.5 \mathrm{~kg}$ & $24(14 \%)$ & $11(10 \%)$ & .46 & $5(7 \%)$ & $7(10 \%)$ & .76 \\
\hline Gestational age (wk) & 38 [37-39] & $38[38-39]$ & .48 & 39 [38-39] & 38 [38-39] & .15 \\
\hline Prenatal diagnosis & $134(80 \%)$ & $79(75 \%)$ & .31 & $50(75 \%)$ & $51(76 \%)$ & .84 \\
\hline Anatomic diagnosis & & & 67 & & & .82 \\
\hline Hypoplastic left heart syndrome & $144(86 \%)$ & $95(90 \%)$ & & $57(85 \%)$ & $59(88 \%)$ & \\
\hline Critical aortic stenosis & $1(1 \%)$ & $1(1 \%)$ & & $1(1 \%)$ & $1(1 \%)$ & \\
\hline Single RV with systemic outflow obstruction & $4(3 \%)$ & $4(4 \%)$ & & $3(4 \%)$ & $3(4 \%)$ & \\
\hline $\begin{array}{l}\text { Right dominant AV canal with systemic outflow } \\
\text { obstruction }\end{array}$ & $11(7 \%)$ & $4(4 \%)$ & & $2(3 \%)$ & $3(4 \%)$ & \\
\hline $\begin{array}{l}\text { Straddling MV with LV hypoplasia and outflow } \\
\text { obstruction }\end{array}$ & $1(1 \%)$ & 0 & & $1(1 \%)$ & 0 & \\
\hline Other & $7(4 \%)$ & $2(2 \%)$ & & $3(4 \%)$ & $1(1 \%)$ & \\
\hline Any associated anatomic diagnosis & $42(25 \%)$ & $22(21 \%)$ & .42 & $19(28 \%)$ & $10(15 \%)$ & .06 \\
\hline Aortic atresia & $110(65 \%)$ & $64(60 \%)$ & .39 & $47(70 \%)$ & $38(57 \%)$ & .11 \\
\hline Obstructed pulmonary venous return & $2(1 \%)$ & $4(4 \%)$ & .16 & 0 & 0 & \\
\hline
\end{tabular}

Data presented as number $(\%)$ or median [interquartile range]. $L-R V P A S$, Left-sided right ventricle-pulmonary artery shunt; $R$ - $R V P A S$, right-sided right ventricle-pulmonary artery shunt; $R V$, right ventricle; $A V$, atrioventricular; $M V$, mitral valve; $L V$, left ventricle. 
TABLE 2. Operative variables of stage I and II procedures

\begin{tabular}{|c|c|c|c|c|c|c|}
\hline \multirow[b]{2}{*}{ Stage I procedure } & \multicolumn{3}{|c|}{ Total cohort } & \multicolumn{3}{|c|}{ Propensity score-matched cohort } \\
\hline & $\begin{array}{c}\text { L-RVPAS } \\
n=168\end{array}$ & $\begin{array}{c}\text { R-RVPAS } \\
\mathbf{n}=106\end{array}$ & $P$ value & $\begin{array}{c}\text { L-RVPAS } \\
n=67\end{array}$ & $\begin{array}{c}\text { R-RVPAS } \\
n=67\end{array}$ & $P$ value \\
\hline Age at Norwood (d) & $6[4-8]$ & $6[5-8]$ & .08 & $6[4-8]$ & $6[5-8]$ & .60 \\
\hline Norwood year & 2006 [2006-2007] & 2006 [2006-2007] & .99 & 2006 [2006-2007] & 2006 [2006-2007] & .94 \\
\hline Center Norwood volume $\leq 15 / y$ & $18(11 \%)$ & $28(26 \%)$ & .001 & $18(27 \%)$ & $15(22 \%)$ & .69 \\
\hline Surgeon Norwood volume $\leq 10 / y$ & $49(27 \%)$ & $56(53 \%)$ & .0001 & $28(42 \%)$ & $35(52 \%)$ & .30 \\
\hline RVPAS diameter (mm) & $5[5-6]$ & $6[5-6]$ & $<.0001$ & $6[5-6]$ & $5[5-6]$ & .44 \\
\hline Indexed RVPAS diameter $(\mathrm{mm} / \mathrm{kg})$ & $1.7[1.5-1.9]$ & $1.8[1.6-2.0]$ & .008 & $1.7[1.5-1.9]$ & $1.8[1.6-2.0]$ & .09 \\
\hline CPB time (min) & $128[96-158]$ & $166[130-185]$ & $<.0001$ & $145[118-174]$ & 147 [118-177] & .77 \\
\hline Crossclamp time (min) & $51[41-61]$ & $66[49-80]$ & $<.0001$ & $55[42-70]$ & $59[45-73]$ & .43 \\
\hline Perfusion strategy & & & $<.0001$ & & & .98 \\
\hline DHCA only & $118(70 \%)$ & $33(31 \%)$ & & $27(40 \%)$ & $28(42 \%)$ & \\
\hline RCP only & $32(19 \%)$ & $43(41 \%)$ & & $24(36 \%)$ & $24(36 \%)$ & \\
\hline DHCA and RCP & $18(11 \%)$ & $30(28 \%)$ & & $16(24 \%)$ & $15(22 \%)$ & \\
\hline Stage II procedure & $\mathbf{n}=132$ & $\mathbf{n}=\mathbf{8 7}$ & & $\mathrm{n}=\mathbf{4 8}$ & $\mathbf{n}=\mathbf{5 9}$ & \\
\hline Age at stage II (d) & 157 [128-196] & 153 [124-183] & .51 & 159 [132-202] & 158 [127-196] & .60 \\
\hline CPB time (min) & 86 [61-127] & 93 [77-133] & .02 & 90 [64-116] & 93 [77-116] & .32 \\
\hline
\end{tabular}

Data presented as number (\%) or median [interquartile range]. $L-R V P A S$, Left-sided right ventricle-pulmonary artery shunt; $R$ - $R V P A S$, right-sided right ventricle-pulmonary artery shunt; $R V P A S$, right ventricle-pulmonary artery shunt; $C P B$, cardiopulmonary bypass; $D H C A$, deep hypothermic circulatory arrest; $R C P$, regional cerebral perfusion.

patients requiring surgical shunt revision was $42 \%$ (5 of 12 deaths), and 15 of the $16(94 \%)$ surgical shunt revisions occurred before Norwood discharge. In the propensity score-matched cohort, similar results were obtained, and the rates of unintended shunt intervention and surgical shunt revision were significantly lower in the R-RVPA shunt group.

\section{Angiographic and Hemodynamic Measurements}

Angiographic and hemodynamic measurements from patients undergoing prestage II catheterization with interpretable angiograms $(n=194)$ are shown in Table 4. Isolated left and right PA diameters were similar between groups in both the total and the propensity score-matched cohorts. There were also no differences in indexed central or peripheral PA measurements, rates of severe branch PA or shunt stenosis, pulmonary-to-systemic flow ratios (Qp:Qs), pulmonary vascular resistance, or arterial oxygen saturations between shunt groups in either cohort.

\section{Perioperative Outcomes and Transplantation-Free Survival}

Perioperative and longitudinal outcomes are shown in Table 5. In the total cohort, there were no significant differences in complications, serious adverse events, death, or cardiac transplantation after operation or at 12 months of follow-up between groups. The rates of phrenic nerve injury $(3.0 \%$ vs $2.3 \%, P=.99)$ and vocal cord injury $(1.5 \%$ vs $0 \%, P=.52$ ) after stage II operation were equivalent between L-RVPA and R-RVPA shunt groups. Kaplan-Meier

TABLE 3. Unintended pulmonary artery and shunt interventions before 12 months of age

\begin{tabular}{|c|c|c|c|c|c|c|}
\hline \multirow[b]{2}{*}{ Intervention } & \multicolumn{3}{|c|}{ Total cohort } & \multicolumn{3}{|c|}{ Propensity score-matched cohort } \\
\hline & $\begin{array}{c}\text { L-RVPAS } \\
(n=168)\end{array}$ & $\begin{array}{c}\text { R-RVPAS } \\
(n=106)\end{array}$ & $P$ value & $\begin{array}{c}\text { L-RVPAS } \\
(n=67)\end{array}$ & $\begin{array}{c}\text { R-RVPAS } \\
(n=67)\end{array}$ & $P$ value \\
\hline \multicolumn{7}{|l|}{ PA interventions } \\
\hline Balloon dilation or stent & $13(7.7)$ & $13(12.3)$ & .33 & $6(9.0)$ & $4(6.0)$ & .75 \\
\hline Surgical augmentation & $56(33.3)$ & $28(26.4)$ & .37 & $23(34.3)$ & $16(23.9)$ & .34 \\
\hline Any PA intervention & $69(41.1)$ & $41(38.7)$ & .84 & $29(43.3)$ & $20(29.9)$ & .25 \\
\hline \multicolumn{7}{|l|}{ Shunt interventions } \\
\hline Balloon dilation or stent & $20(11.9)$ & $9(8.5)$ & .52 & $7(10.4)$ & $7(10.4)$ & .99 \\
\hline Surgical revision & $14(8.3)$ & $2(1.9)$ & .05 & $12(17.9)$ & 0 & $<.001$ \\
\hline Conversion to MBTS & $3(1.8)$ & $3(2.8)$ & .86 & $2(3.0)$ & $2(3.0)$ & .99 \\
\hline Any shunt intervention & $37(22.0)$ & $14(13.2)$ & .12 & $21(31.3)$ & $9(13.4)$ & .04 \\
\hline
\end{tabular}


TABLE 4. Prestage II catheterization pulmonary artery and hemodynamic measurements

\begin{tabular}{|c|c|c|c|c|c|c|}
\hline \multirow[b]{2}{*}{ Variable } & \multicolumn{3}{|c|}{ Total cohort } & \multicolumn{3}{|c|}{ Propensity score-matched cohort } \\
\hline & $\begin{array}{l}\text { L-RVPAS } \\
(n=114)\end{array}$ & $\begin{array}{c}\text { R-RVPAS } \\
(\mathbf{n}=\mathbf{8 0})\end{array}$ & $P$ value & $\begin{array}{c}\text { L-RVPAS } \\
(n=45)\end{array}$ & $\begin{array}{c}\text { R-RVPAS } \\
(\mathbf{n}=\mathbf{5 4})\end{array}$ & $P$ value \\
\hline Body surface area $\left(\mathrm{m}^{2}\right)$ & $0.31[0.28-0.34]$ & $0.31[0.30-0.34]$ & .13 & $0.31[0.29-0.34]$ & $0.32[0.30-0.34]$ & .39 \\
\hline \multicolumn{7}{|l|}{ Central PA measurements } \\
\hline Mid-main left PA diameter (mm) & $4.2[3.4-5.2]$ & $4.8[3.6-5.8]$ & .16 & $4.4[3.6-5.4]$ & $4.8[3.6-6.2]$ & .72 \\
\hline Mid-main right PA diameter $(\mathrm{mm})$ & $4.1[3.2-5.1]$ & $4.4[3.6-5.6]$ & .12 & $4.7[3.6-5.9]$ & $4.5[3.8-5.6]$ & .80 \\
\hline PA (Nakata) index $\left(\mathrm{mm}^{2} / \mathrm{m}^{2}\right)$ & $91[59-135]$ & $118[72-158]$ & .05 & 106 [73-169] & $121[73-162]$ & .73 \\
\hline \multicolumn{7}{|l|}{ Peripheral PA measurements } \\
\hline Proximal left lower lobe diameter (mm) & $4.9[4.2-6.3]$ & $4.8[4.2-5.7]$ & .29 & $4.5[4.2-6.2]$ & $5.0[4.0-5.8]$ & .87 \\
\hline Proximal right lower lobe diameter $(\mathrm{mm})$ & $4.8[4.1-5.8]$ & $5.2[4.2-5.9]$ & .28 & $5.1[4.5-6.0]$ & $5.2[4.5-5.9]$ & .75 \\
\hline Total lower lobe index $\left(\mathrm{mm}^{2} / \mathrm{m}^{2}\right)$ & 135 [100-193] & $134[100-164]$ & .41 & $150[99-178]$ & $136[99-179]$ & .57 \\
\hline \multicolumn{7}{|l|}{ Stenoses } \\
\hline Severe left PA stenosis $(>50 \%)$ & $8(7 \%)$ & $5(6 \%)$ & .83 & $1(2 \%)$ & $4(7 \%)$ & .24 \\
\hline Severe right PA stenosis $(>50 \%)$ & $13(11 \%)$ & $9(11 \%)$ & .97 & $6(13 \%)$ & $5(9 \%)$ & .52 \\
\hline Shunt stenosis & $31(28 \%)$ & $23(29 \%)$ & .87 & $10(23 \%)$ & $20(38 \%)$ & .11 \\
\hline \multicolumn{7}{|l|}{ Hemodynamic measurements } \\
\hline Aortic oxygen saturation $(\%)$ & $74[69-78]$ & 75 [71-78] & .29 & $76[68-78]$ & 75 [71-79] & .51 \\
\hline Pulmonary-to-systemic flow ratio (Qp:Qs) & $1[0.8-1.4]$ & $1[0.7-1.1]$ & .05 & $1.2[0.9-1.5]$ & $1.0[0.7-1.3]$ & .09 \\
\hline PVR (Wood units) & 1.9 [1.3-2.9] & $2.0[1.6-2.8]$ & .52 & $2.3[1.3-2.9]$ & $2.0[1.7-3.0]$ & .83 \\
\hline
\end{tabular}

Data presented as number (\%) or median [interquartile range]. $L$-RVPAS, Left-sided right ventricle-pulmonary artery shunt; $R$-RVPAS, right-sided right ventricle-pulmonary artery shunt; $P A$, pulmonary artery; $P V R$, pulmonary vascular resistance.

analysis similarly demonstrated no difference in transplantation-free survival between L-RVPA and RRVPA shunt groups at 3 years of follow-up (Figure 1, A).

In the propensity score-matched cohort, patients in the RRVPA shunt group experienced fewer serious adverse events at 12 months of follow-up and improved transplantation-free survival at 36 months of follow-up (LRVPA: 61\% [95\% confidence interval, 48-72], R-RVPA: 80\% [95\% confidence interval, 69-88]) (Figure 1, B).

\section{DISCUSSION}

The SVR trial identified a short-term survival advantage with use of RVPA shunts during the Norwood procedure but also revealed several limitations of "first-generation" RVPA shunts, including increased shunt and PA interventions and worse PA growth. ${ }^{2}$ Since then, several technical modifications have been conceived in an attempt to mitigate the shortcomings of RVPA shunts while maintaining the hemodynamic advantages. ${ }^{1}$ We examined RVPA shunt position relative to the neoaorta in the SVR trial public use dataset and found that RVPA shunt placement to the right of the neoaorta was associated with fewer surgical shunt revisions compared with L-RVPA shunt placement and may lead to improved survival in select patients. However, other key end points, including PA size, PA reinterventions, and hemodynamic parameters, appeared unaffected by RVPA shunt position.

TABLE 5. Perioperative and 12-month outcomes

\begin{tabular}{|c|c|c|c|c|c|c|}
\hline \multirow[b]{2}{*}{ Stage I outcomes } & \multicolumn{3}{|c|}{ Total cohort } & \multicolumn{3}{|c|}{ Propensity score-matched cohort } \\
\hline & $\begin{array}{l}\text { L-RVPAS } \\
(\mathrm{n}=168)\end{array}$ & $\begin{array}{l}\text { R-RVPAS } \\
(\mathbf{n}=106)\end{array}$ & $P$ value & $\begin{array}{l}\text { L-RVPAS } \\
(n=67)\end{array}$ & $\begin{array}{c}\text { R-RVPAS } \\
(\mathbf{n}=67)\end{array}$ & $P$ value \\
\hline Complications, No. (rate/100 infants) & $474(282)$ & $267(252)$ & .15 & $212(316)$ & $166(248)$ & .02 \\
\hline Discharged alive, No. (\%) & $146(87 \%)$ & $91(86 \%)$ & .80 & $55(82 \%)$ & $61(91 \%)$ & .13 \\
\hline Stage II outcomes & $\mathbf{n}=\mathbf{1 3 2}$ & $\mathbf{n}=\mathbf{8 7}$ & & $\mathrm{n}=\mathbf{4 8}$ & $\mathbf{n}=\mathbf{5 9}$ & \\
\hline Complications, No. (rate/100 infants) & $148(112)$ & $119(137)$ & .12 & $37(77)$ & $75(127)$ & .01 \\
\hline Discharged alive, No. $(\%)$ & $125(95 \%)$ & $83(95 \%)$ & .82 & $47(98 \%)$ & $56(95 \%)$ & .42 \\
\hline Stage 1 operation to $12 \mathrm{mo}$ & $\mathrm{n}=168$ & $\mathbf{n}=106$ & & $\mathrm{n}=67$ & $\mathrm{n}=67$ & \\
\hline Complications, No. (rate/100 infants) & $877(522)$ & $531(501)$ & .47 & $344(513)$ & $330(493)$ & .62 \\
\hline $\begin{array}{l}\text { Serious adverse events, No. } \\
\text { (rate/100 infants) }\end{array}$ & $101(60)$ & $52(49)$ & .27 & $56(84)$ & $31(46)$ & .01 \\
\hline Death or cardiac transplantation & $44(26 \%)$ & $24(23 \%)$ & .51 & $18(27 \%)$ & $12(18 \%)$ & .21 \\
\hline
\end{tabular}

$L-R V P A S$, Left-sided right ventricle-pulmonary artery shunt; $R$-RVPAS, right-sided right ventricle-pulmonary artery shunt. 

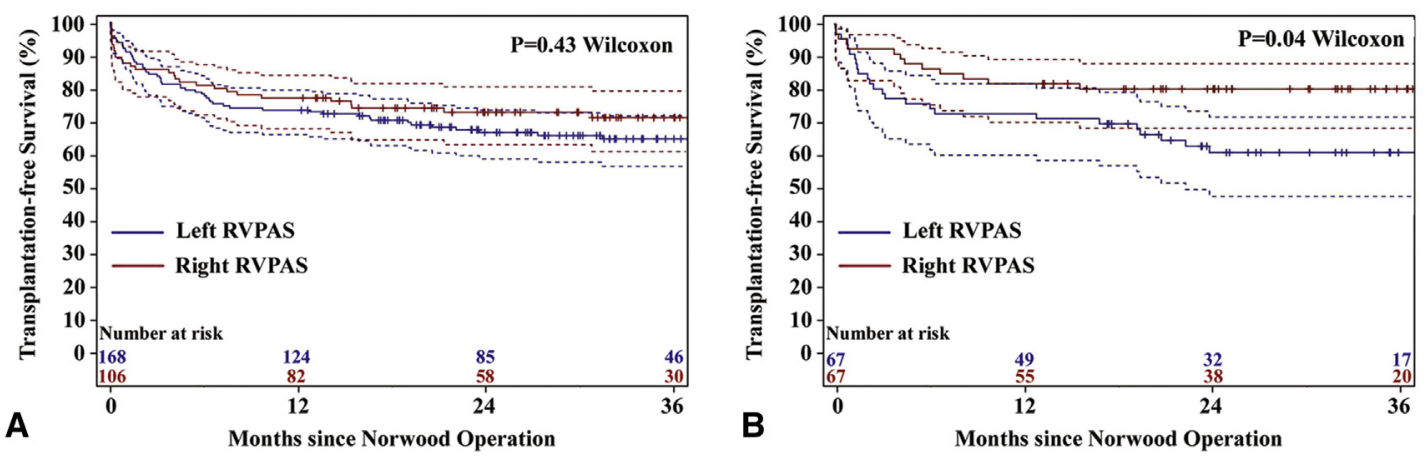

FIGURE 1. Transplantation-free survival stratified by shunt position in the (A) total cohort and (B) propensity score-matched cohort. RVPAS, Right ventricle-pulmonary artery shunt.

\section{Shunt Interventions}

Construction of an RVPA shunt is technically challenging and has been the topic of considerable inquiry and debate. Technical considerations that require careful attention include (1) creation of the ventriculotomy and proximal anastomoses to prevent dynamic obstruction from ventricular muscle; (2) attention to the length, lie, and course of the shunt to prevent bending or kinking of the mid-portion or neoaorta/coronary compression; and (3) avoidance of tension or kinking at the site of distal PA insertion to prevent distal shunt stenosis or "bowtie" PA deformity. Presumably as a result of these technical challenges, the SVR trial and other single-institution series have reported an increased need for shunt intervention with use of RVPA shunts. ${ }^{2,12}$ We found a reduced rate of surgical shunt revision and overall shunt interventions with R-RVPA shunts when compared with LRVPA shunts. Although the SVR trial data preclude a more granular analysis of the cause and nature of these shunt interventions, it should be noted that $94 \%$ of the surgical shunt revisions were performed during the Norwood hospitalization, whereas the rate of angiographic shunt stenosis observed later at prestage II catheterization was equivalent between groups ( $28 \%$ vs $29 \%$ ). Thus, the data suggest there were likely fewer technical issues with R-RVPA shunt placement requiring early surgical revision, perhaps in part due to an improved lie of the shunt with less kinking or distortion at the mid-portion or proximal and distal insertion sites. ${ }^{4}$ Although it remains possible that other technical aspects of RVPA shunt creation not assessed in this dataset may partially explain the improved results in the R-RVPA shunt group, it should be noted that the first published reports advocating the use of ring-reinforced conduits $^{13}$ or the proximal $^{14}$ or distal ${ }^{15}$ dunk technique appeared well after the time period of the SVR trial, suggesting that these additional technical modifications may have been less relevant during the SVR trial period.

\section{Pulmonary Artery Interventions and Size}

The SVR trial and other nonrandomized studies reported increased rates of PA interventions with use of RVPA shunts compared with MBT shunts, typically because of central PA stenosis at the site of RVPA shunt insertion ("bowtie" deformity) thought to result from tension or kinking at the distal anastomosis or increased shear stress and neointimal proliferation from pulsatile flow. ${ }^{2,5-7,12}$ As a result, central PA augmentation is required in as much as $80 \%$ of RVPA shunt cases at the time of stage II operation. ${ }^{5}$ However, data regarding the impact of RVPA shunts on branch PA growth are mixed. Some centers have reported improved branch PA growth with RVPA shunts, ${ }^{4,7,12}$ presumably because of pulsatile flow and the more equal distribution of pulmonary blood flow compared with the predominantly right-sided MBT shunt placement. In contrast, the SVR trial identified worse central PA growth with RVPA shunts, as evidenced by a lower Nakata index and a higher rate of severe left branch PA stenosis. ${ }^{2,3}$ Previous comparisons of PA interventions and growth between L- and R-RVPA shunts in small single-institution studies also have produced conflicting results. ${ }^{5-7}$

In our analysis of the SVR trial, we found no difference in the overall rate of PA intervention, angiographic PA size, or branch PA stenosis at prestage II catheterization between R-RVPA and L-RVPA shunt cases, suggesting that RVPA shunt position had little impact on the development of central or branch PA stenosis. These data suggest that other technical modifications aside from shunt position are needed to reduce the rate of PA stenosis with RVPA shunts. ${ }^{15}$

\section{Perioperative and Long-Term Outcomes}

Prior authors have advocated for R-RVPA shunt placement because of greater ease of surgical access to the distal shunt and central PAs during the stage II procedure, avoiding the difficult dissection of the central PAs behind the neoaorta with the attendant risks of phrenic and recurrent laryngeal nerve injury and increased operative times. ${ }^{4,5,7}$ Conversely, concerns with R-RVPA shunt placement include the possibility of shunt compression of the coronary arteries or neoaortic root, as well as damage to the shunt during sternal reentry due to a retrosternal shunt trajectory that crosses the midline. ${ }^{6}$ Last, long-term 
survival beyond the stage II procedure may be affected if shunt position leads to improved Fontan candidacy because of superior pulmonary blood flow and lung development. ${ }^{5}$

In the overall patient cohort, our analysis of the SVR trial failed to identify any objective differences in perioperative outcomes associated with RVPA shunt position. Specifically, there were no differences in complications, serious adverse events, nerve injury, or discharge mortality after the stage I or II operations. Paradoxically, CBP and crossclamp times were longer with the R-RVPA shunt position. However, these results were heavily confounded by the differences in DHCA perfusion strategy, which essentially invalidates the comparison of perfusion times between groups. Transplantation-free survival at 3 years was further equivalent between RVPA shunt groups. However, these comparisons of patient outcomes in the overall cohort may have been partially confounded by differences in operative parameters, such as surgeon and center volume, which may have favored improved outcomes in the L-RVPA group ${ }^{16,17}$ and masked any benefit of R-RVPA shunt position.

In the propensity score-matched cohort with balanced operative parameters, the results demonstrated fewer serious adverse events at 12 months and improved transplantation-free survival at 3 years in patients with RRVPA shunts, despite the slightly lower birth weight of these patients. Surgical shunt revisions were associated with a $42 \%$ mortality rate and coincided with several serious adverse events, including death, acute shunt failure, cardiac arrest, extracorporeal membrane oxygenation, and unplanned cardiovascular reoperation. Thus, in the absence of other major differences in surgeon or center practices that could affect outcomes, it remains mechanistically feasible that the improved results in the propensity score-matched R-RVPA shunt group were related to fewer surgical shunt revisions. However, given that the propensity score match excluded more than $50 \%$ of patients, including the majority of patients who underwent surgery with DHCA, these results may not be generalizable to the overall Norwood population, and we view these survival results as speculative and hypothesis generating at this time.

\section{Study Limitations}

Our study contains several important limitations that should be acknowledged. RVPA shunt position was a nonrandomized surgical variable that was dictated by surgeon preference. Propensity score matching was used in an attempt to normalize the major differences in operative parameters between groups. However, the propensity score match excluded the majority of patients who underwent surgery with DHCA and yielded differences in birth weight between groups. Therefore, the results of the propensity score match subgroup analysis may not be generalizable to the population with circulatory arrest. The slightly lower birth weight of the patients in the R-RVPA group may have predisposed them to worse outcomes given that birth weight is a risk factor for morbidity and mortality after the Norwood operation, although there was no difference in the highest risk group of neonates with birth weights less than $2.5 \mathrm{~kg} .{ }^{16,18}$ In addition, shunt position may serve as a marker for several other surgeon preferences, center differences, or technical differences in shunt creation that were unrecorded in this dataset and unable to be controlled for by propensity matching. Further, other important late end points that may be influenced by pulmonary development, such as quality of life, exercise capacity, and functional capacity through the Fontan operation, were not assessed in this dataset. Finally, the surgical techniques of RVPA shunt creation and outcomes achieved during the study period from 2005 to 2008 may represent the learning curve for some surgeons and may not be reflective of contemporary practices.

\section{CONCLUSIONS}

In this unplanned secondary analysis of the SVR trial public use dataset, we found that RVPA shunt placement to the right of the neoaorta was associated with fewer shunt revisions and improved survival in a propensity score-matched cohort, suggesting that R-RVPA shunt placement may partially alleviate some of the previously recognized limitations of RVPA conduits. However, other key end points, including PA size, PA reinterventions, and hemodynamic parameters, appeared unaffected by RVPA shunt position.

\section{Conflict of Interest Statement}

Authors have nothing to disclose with regard to commercial support.

The authors thank Sally Cai for assistance with statistical revisions.

\section{References}

1. Jaquiss RD. Hypoplastic left heart syndrome: how to improve late survival and quality of life. J Thorac Cardiovasc Surg. 2015;150:1401-3.

2. Ohye RG, Sleeper LA, Mahony L, Newburger JW, Pearson GD, Lu M, et al. Comparison of shunt types in the Norwood procedure for single-ventricle lesions. N Engl J Med. 2010;362:1980-92.

3. Aiyagari R, Rhodes JF, Shrader P, Radtke WA, Bandisode VM, Bergersen L, et al. Impact of pre-stage II hemodynamics and pulmonary artery anatomy on 12-month outcomes in the Pediatric Heart Network Single Ventricle Reconstruction trial. J Thorac Cardiovasc Surg. 2014;148:1467-74.

4. Rumball EM, McGuirk SP, Stumper O, Laker SJ, de Giovanni JV, Wright JG, et al. The RV-PA conduit stimulates better growth of the pulmonary arteries in hypoplastic left heart syndrome. Eur J Cardiothorac Surg. 2005;27:801-6.

5. Barron DJ, Brooks A, Stickley J, Woolley SM, Stumper O, Jones TJ, et al. The Norwood procedure using a right ventricle-pulmonary artery conduit: comparison of the right-sided versus left-sided conduit position. J Thorac Cardiovasc Surg. 2009;138:528-37.

6. Kolcz J, Skladzien T, Kordon Z, Rudzinski A, Skalski J. Impact of right ventriclepulmonary artery conduit placement on pulmonary artery development after the Norwood procedure in hypoplastic left heart syndrome. Eur J Cardiothorac Surg. 2012;42:218-24.

7. Gist KM, Barrett CS, Graham DA, Crumback SL, Schuchardt EL, Erickson B, et al. Pulmonary artery interventions after Norwood procedure: does type or 
position of shunt predict need for intervention? J Thorac Cardiovasc Surg. 2013; 145:1485-92.

8. Ohye RG, Gaynor JW, Ghanayem NS, Goldberg CS, Laussen PC, Frommelt PC, et al. Design and rationale of a randomized trial comparing the Blalock-Taussig and right ventricle-pulmonary artery shunts in the Norwood procedure. $J$ Thorac Cardiovasc Surg. 2008;136:968-75.

9. Hill KD, Rhodes JF, Aiyagari R, Baker GH, Bergersen L, Chai PJ, et al. Intervention for recoarctation in the single ventricle reconstruction trial: incidence, risk, and outcomes. Circulation. 2013;128:954-61.

10. Ovroutski S, Ewert P, Alexi-Meskishvili V, Holscher K, Miera O, Peters B, et al. Absence of pulmonary artery growth after Fontan operation and its possible impact on late outcome. Ann Thorac Surg. 2009;87:826-31.

11. Nakata S, Imai Y, Takanashi Y, Kurosawa H, Tezuka K, Nakazawa M, et al. A new method for the quantitative standardization of cross-sectional areas of the pulmonary arteries in congenital heart diseases with decreased pulmonary blood flow. J Thorac Cardiovasc Surg. 1984;88:610-9.

12. Pruetz JD, Badran S, Dorey F, Starnes VA, Lewis AB. Differential branch pulmonary artery growth after the Norwood procedure with right ventricle-pulmonary artery conduit versus modified Blalock-Taussig shunt in hypoplastic left heart syndrome. J Thorac Cardiovasc Surg. 2009;137:1342-8.

13. Bentham JR, Baird CW, Porras DP, Rathod RH, Marshall AC. A reinforced rightventricle-to-pulmonary-artery conduit for the stage-1 Norwood procedure improves pulmonary artery growth. J Thorac Cardiovasc Surg. 2015;149: 1502-8.e1.

14. Tweddell JS, Mitchell ME, Woods RK, Spray TL, Quintessenza JA. Construction of the right ventricle-to-pulmonary artery conduit in the Norwood: the "dunk" technique. Oper Tech Thorac Cardiovasc Surg. 2012;17:81-98.

15. Mascio CE, Spray TL. Distal dunk for right ventricle to pulmonary artery shunt in stage 1 palliation. Ann Thorac Surg. 2015;100:2381-2.

16. Tabbutt S, Ghanayem N, Ravishankar C, Sleeper LA, Cooper DS, Frank DU, et al. Risk factors for hospital morbidity and mortality after the Norwood procedure: a report from the Pediatric Heart Network Single Ventricle Reconstruction trial. J Thorac Cardiovasc Surg. 2012;144:882-95.

17. Pasquali SK, Jacobs JP, He X, Hornik CP, Jaquiss RD, Jacobs ML, et al. The complex relationship between center volume and outcome in patients undergoing the Norwood operation. Ann Thorac Surg. 2012;93:1556-62.

18. Curzon CL, Milford-Beland S, Li JS, O’Brien SM, Jacobs JP, Jacobs ML, et al. Cardiac surgery in infants with low birth weight is associated with increased mortality: analysis of the Society of Thoracic Surgeons Congenital Heart Database. J Thorac Cardiovasc Surg. 2008;135:546-51.

Key Words: congenital heart surgery, Norwood procedure, hypoplastic left heart syndrome, Single Ventricle Reconstruction trial

\section{Discussion}

Dr C. Selzman (Salt Lake City, Utah). The last 2 articles were both Sampson Award candidates, as is this next one, which is from Nicholas Andersen and colleagues from Duke University, "A Comparison of Right Ventricular Pulmonary Artery Shunt Position and the Norwood Procedure: An Analysis of the Pediatric Heart Network Public Database."

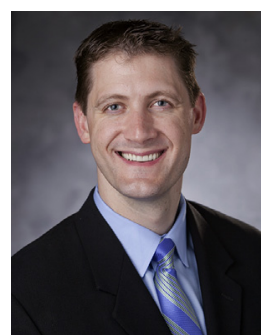

Dr N. Andersen (Durham, NC). The Pediatric Heart Network SVR Trial evaluated the source of pulmonary blood flow in patients undergoing the Norwood procedure and demonstrated that RVPA shunts led to improved early survival compared with MBT shunts but came at the expense of more unintended interventions and worse PA growth. However, numerous technical aspects of RVPA shunt placement may further influence these results. One technical variable that was recorded in the SVR trial dataset was placement of the shunt to the left or right of the neoaorta. Several small single institution studies have reported improved outcomes with the R-RVPA shunt.

Reported benefits of rightward shunt placement include greater ease of future surgical access to the distal shunt by avoiding the difficult dissection of the shunt behind the neoaorta and thereby leading to reduced rates of phrenic and recurrent laryngeal nerve injury and shorter operative and perfusion times during the stage II operation. Other reported benefits include reduced kinking of the shunt leading to fewer shunt reinterventions and reduced tension on the central PAs and more symmetric PA blood flow leading to improved PA growth and fewer PA reinterventions. We aimed to validate these findings in a larger multi-institutional patient cohort and hypothesized that R-RVPA shunt placement would be associated with superior outcomes. The Pediatric Heart Network SVR Trial public use dataset was used for our analysis. The SVR trial was conducted between 2005 and 2008 at 15 North American centers and randomized 549 patients with single right ventricle lesions to receive an RVPA shunt or MBT shunt during the Norwood procedure. A total of 281 patients underwent RVPA shunt placement at the conclusion of the Norwood procedure, and the position of the shunt relative to the neoaorta was recorded for 274 patients who comprised our final study cohort.

Baseline characteristics, operative parameters, perioperative outcomes, transplantation-free survival, reinterventions, and angiographic measurements were then compared between patients who received an L- or R-RVPA shunt. Results showed that left-sided shunts were used more commonly and placed in $61 \%$ of patients, whereas right-sided shunts were placed in $39 \%$ of patients. The proportion of left- and right-sided shunts remained nearly constant from year to year, suggesting no change in surgeon preference for shunt position over the course of the trial. Although RVPA shunt position was not randomized, there were no significant differences in baseline patient characteristics between leftand right-sided shunt groups, including birth weight, gestational age, chromosomal or anatomic abnormalities, and cardiac diagnosis. As a result, risk adjustment of outcomes was not performed, and all associations presented in the remainder of this study are unadjusted. Differences in operative parameters were appreciated between groups. Left-sided shunts were used more commonly by high-volume centers and surgeons, with the highest volume placing exclusively left-sided shunts. 
CPB and crossclamp times were 38 and 15 minutes longer, respectively, with the use of right-sided shunts; however, the use of circulatory arrest as the perfusion strategy was twice as common in the left-sided shunt cases, confounding the comparison of bypass times between groups. Shunt diameters also differed between groups, with 5-mm shunts used most commonly in the left-sided shunt group and 6-mm shunts used more often in the right-sided shunt group. Perioperative outcomes after the Norwood operation were equivalent, and there were no differences in total complications or survival to hospital discharge between the left- and right-sided shunt groups. Approximately $80 \%$ of patients in each group progressed to the stage 2 operation. Cardiopulmonary bypass times at the stage 2 operation were $7 \mathrm{mi}-$ nutes longer with right-sided shunts, although again, there was a 5-fold increase in the use of circulatory arrest with left-sided shunts that confounded the comparison of bypass times. Perioperative outcomes after the stage 2 procedure were equivalent between groups, and there were no differences in total complications, phrenic or recurrent laryngeal nerve injury, or survival to hospital discharge after the stage 2 procedure.

Kaplan-Meier analysis also demonstrated no difference in transplantation-free survival between the Land R-RVPA shunt groups at 3 years of follow-up, suggesting that shunt position did not affect procedural or midterm survival outcomes. Unintended PA reinterventions were recorded for the first 12 months after the Norwood operation. During follow-up, there were no differences in the rates of catheter-based or surgical PA interventions between left- and right-sided shunt cases, including surgical pulmonary arterioplasty at the time of the stage 2 operation. Contrary to prior reports, these data suggest that R-RVPA shunt position did not affect the rate of central or branch PA stenosis requiring intervention. Unintended shunt reinterventions also were recorded for the first 12 months after the Norwood operation. The total rate of unintended catheter-based or surgical shunt interventions was $40 \%$ lower with R-RVPA shunts, although this difference did not reach statistical significance; however, the rate of isolated surgical shunt revision was 4-fold lower with right-sided shunts.

Of note, $94 \%$ of these surgical shunt revisions were early events that occurred during the Norwood hospitalization. Thus, these data suggest there may have been fewer technical issues with R-RVPA shunts requiring early surgical revision, perhaps in part because of an improved lie of the shunt with less kinking or distortion at the insertion sites. Angiographic and hemodynamic measurements were recorded from patients who underwent prestage 2 catheterization. The Nakata index was used to estimate central PA growth from mid main branch PA measurements, and the lower lobe index was used to estimate peripheral PA growth from the proximal lower lobe branch PAs. There was a trend toward larger central PA diameters with right-sided shunts, and the indexed sum of the central PA areas was 30\% larger in the right-sided shunt group, potentially supporting the theory of more equal distribution of pulmonary blood flow from a more central shunt location or improved angulation of the shunt. However, the peripheral PA diameters and the indexed lower lobe branch PA areas were equivalent between groups.

Finally, hemodynamic measurements demonstrated similar arterial oxygen saturations, pulmonary to systemic flow ratios, and pulmonary vascular resistance between groups, suggesting similar pulmonary blood flow between shunt positions. This study contains several important limitations. The RVPA shunt position was not randomized and was dictated by surgeon preference. The outcomes between groups were unadjusted because of the small number of centers, patients, and events, and the similar baseline characteristics between groups. However, shunt position may serve as a marker for several other unmeasured surgeon preferences, center differences, or technical differences in shunt creation that may account for these results. Differences in surgeon and center volume may have masked any advantage of R-RVPA shunts given that they were preferentially used by surgeons with a lower volume of operations and centers where baseline outcomes may be expected to be worse. The surgical techniques of RVPA shunt creation used during the study period may represent the learning curve for some surgeons and not reflect contemporary practices.

Important late end points that may be influenced by pulmonary development, such as quality of life, exercise capacity, and functional survival through the Fontan operation, were not assessed in this dataset. In this secondary analysis of the SVR trial public use dataset, we found that RVPA shunt placement to the right of the neoaorta was associated with fewer shunt revisions, and improved central PA growth, suggesting that RRVPA shunt placement may partially alleviate some of the previously recognized shortcomings of RVPA conduits. However, other key end points were unaffected by RVPA shunt location, including PA reinterventions, perioperative outcomes, and transplantation-free survival.

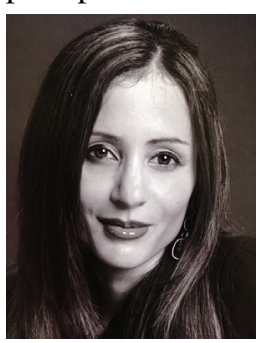

Dr T. Karamlou (San Francisco, Calif). Andersen and colleagues have used the SVR public use dataset to investigate the impact of RV-PA conduit position relative to the neoaorta on both short- and long-term outcomes. The authors compared 168 leftsided shunts with 106 right-sided shunts and found that right-sided shunts were associated 
with a lower prevalence of surgical shunt revision within 12 months of Norwood reconstruction and an increased Nakata index in the prestage 2 cardiac catheter cases. Although the authors found 2 reported benefits of the right-sided conduit, the lack of other clinically meaningful differences between the groups does limit the import to their findings. I have the following 3 questions and 1 comment for the authors. Number one, although as you mentioned, the SVR trial was randomized, the randomization piece refers to triage to a Blalock-Taussig shunt or an RV-PA conduit and, therefore, does not apply to a comparison between groups regarding the shunt position. The authors, unfortunately, performed only an unadjusted comparison stating that baseline characteristics did not differ between the groups. The flaw in this rationale is that one cannot know whether uncompared baseline or operative factors have biased the findings; therefore, risk adjustment is critical and cannot be omitted. Can the authors comment on whether they plan to do a multivariable analysis to address this issue?

Dr Andersen. Your comments really get to the heart of this study quite well. First, I want to emphasize as you have that this variable we looked at was not randomized even though it came from a randomized controlled trial, and so people should not be fooled into thinking that these data are any stronger than your typical retrospective study. The strength of this dataset is that it contains prospectively collected high-quality data from several institutions, but the study still contains the same weaknesses of any retrospective study of selection bias and unmeasured confounders. Our findings also showed that the 2 shunt groups were more similar than different, which is actually in contrast to some of the previous articles on this topic. So I don't think any surgeon should watch this presentation and walk away thinking that they're doing something wrong or they need to switch and do things differently. The results show more similarities than differences between these 2 groups. As far as risk adjustment, this is something we thought about a lot. What we had were 2 groups of patients who had similar baseline characteristics. One of the goals of risk adjustment is, of course, to normalize baseline risk going into the intervention. Baseline characteristics were fairly equivalent. There were differences in center volume, which has been associated with outcomes after the Norwood procedure, so the best thing to adjust for probably would be center volume, and here the math just kind of fell apart. There were only 15 centers in the SVR trial, and in the public use dataset, they don't tell you the center identity or the actual volume of each center. They just place the 15 centers into 4 arbitrary volume categories. Now, you have a categorical variable divided into 4 baskets, and each basket contains only 2 to 3 centers. Using that variable to adjust for outcomes with a small number of events, such as shunt reintervention where there was only 16 events in the entire study, is problematic. Our statisticians looked at this dataset, and we spoke at length, and they basically told me we can do the risk adjustment, but we don't think it's going to be valid, and so we held back. I think the more important issue though is the one you mentioned about unmeasured confounders. There's clearly going to be unmeasured confounders because there are distinct surgeon signatures embedded within this dataset. There were clearly high-volume surgeons who preferred to use leftsided shunts and circulatory arrest and mid- to low-volume surgeons who used right-sided shunts and regional perfusion. Clearly, there's going to be other technical differences embedded within those surgeon signatures that we can't measure, which I think would include things such as use of ring-reinforced conduits, proximal dunk technique, and so forth. Some of these other things may be hiding in the data that were not recorded, which clearly could be influencing the results.

Dr Karamlou. I would stress risk adjustment is going to be critical in the review process. Number 2, it appears that the 6-mm conduit was more commonly used on the rightsided conduit group, $52 \%$ compared with $26 \%$. The authors stated that this should not have influenced their findings that central PA growth was better in the right-sided group because of the lack of difference in the QP:Qs, PVR, and so forth among the groups. Can the authors comment on the reasons that peripheral pulmonary arterial growth was equal among groups despite a suggested unequal flow distribution in the left-sided conduits. Were central PA reconstructions more commonly used among the left-sided group? In other words, is this growth discrepancy clinically significant?

Dr Andersen. There was absolutely no difference in PA interventions between groups and no difference in the peripheral PA measurements. The only difference was the central PA measurements. If we believe that is a true finding, the best explanation is that it might have something to do with turbulence of blood flow at the distal insertion site, which could be affecting the central PA growth but not translating into any differences in peripheral PA growth.

Dr Karamlou. Finally, you've already addressed the issue about surgeon and center volume. My final comment, included in your article, but not in your presentation, deals with complexity. I do not agree with the statement in the article that the construction of an MBT shunt is less complex than that of an RV to PA conduit. In fact, the Society of Thoracic Surgeons stat category is 4 for a Blalock-Taussig shunt compared with 2 for an RVA PA conduit, reflecting both empiric and consensus-based agreement with my presumption. I would consider deleting that comment from your article.

Dr Andersen. Thank you.

Dr M. Mulligan (Seattle, Wash). I agree with Tara. There are some flaws, and maybe they are just flaws in the study, 
but in many cases, the choice of shunt depends on the surgeon and the anatomy. These are difficult things to pull out of these data. For instance, doubling the types of single ventricular heart disease often are best treated with a rightsided shunt, whereas a garden-variety Norwood procedure is better taken care of with a left-sided shunt. That's my personal bias, and so as one surgeon of many, my own data probably would confound this because I will change my procedure depending on what I see in the operating room.

Dr Andersen. Sure.

Dr Mulligan. One of the limitations is that it would be difficult to delve into what's going on in a surgeon's mind at the time of the operation. I agree with Tara that this is a nice descriptive article, but it would be difficult to use these data to change one's clinical practice.

Dr Andersen. I have 2 comments that come to mind. One is that there are articles showing that right-sided shunts lead to improved survival and fewer reinterventions. One interpretation of our study is that it may provide some stronger data showing that the 2 shunt techniques are more similar than different. The other comment I'll make is that you're absolutely right that this is a difficult topic to study. We've taken a technical question and tried to answer it in a multicenter database, which is almost never done. Generally, when you hear about a new technical advance or idea, it's generally a single surgeon at a single center who tries something in 10 patients and says, "Hey, this worked great," and he or she writes an article and compares the results with their last 10 patients. That's how we learn about technical advances. To try to ask a technical question in a multicenter prospective database is a good idea, but it's very difficult, and I think this study and your comments highlight where the data break down. But again, I think you can interpret this study as showing that in a large multicenter database the 2 shunt groups were more similar than different, and there were actually few objective differences between the shunt groups. 


\section{Distribution of Propensity}
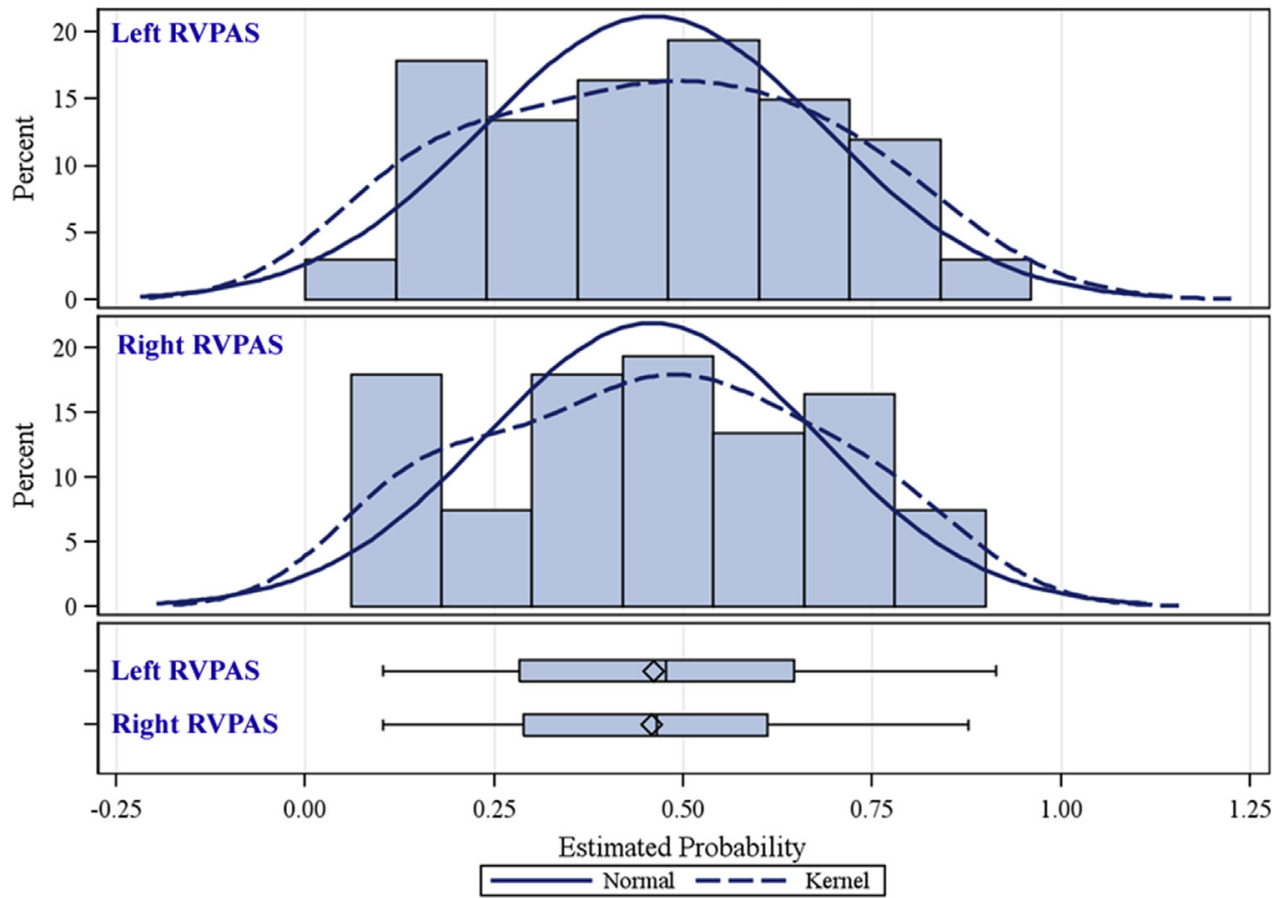

FIGURE E1. Distribution of propensity scores for matched patients. RVPAS, Right ventricle-pulmonary artery shunt.

TABLE E1. Matching model results

\begin{tabular}{lc}
\hline \multicolumn{1}{c}{ Variable } & Propensity score-matched cohort \\
\cline { 2 - 3 } & $\mathbf{n = 6 7}$ per group \\
\hline Male sex & .054 \\
Birth weight (kg) & .451 \\
Gestational age (wk) & .228 \\
Prenatal diagnosis & .096 \\
Any associated anatomic diagnosis & .118 \\
Age at Norwood (d) & .287 \\
Norwood year & .155 \\
Center Norwood volume $\leq 15 / y$ & .250 \\
RVPAS diameter (mm) & .089 \\
CPB time (stage 1, min) & .071 \\
Crossclamp time (stage 1, min) & .239 \\
Perfusion strategy (stage 1) & .092 \\
Data are shown as standardized difference of the mean. RVPAS, Right ventricle-pul- \\
monary artery shunt; CPB, cardiopulmonary bypass.
\end{tabular}

